\title{
A Novel In Silico Method for Molecular Mimicry Detection Finds a Formin with the Potential to Manipulate the Maize Cell Cytoskeleton
}

\author{
Vinicio Armijos-Jaramillo, ${ }^{1,2, \dagger}$ Nicole Espinosa, ${ }^{1}$ Karla Vizcaíno, ${ }^{1}$ and Daniela Santander-Gordón ${ }^{1}$ \\ ${ }^{1}$ Carrera de Ingeniería en Biotecnología, Facultad de Ingeniería y Ciencias Aplicadas, Universidad de Las Américas, Quito, \\ Ecuador \\ ${ }^{2}$ Grupo de Bio-Quimioinformática, Universidad de Las Américas, Quito, Ecuador
}

Accepted 21 March 2021.

\begin{abstract}
Molecular mimicry is one of the evolutionary strategies that parasites use to manipulate the host metabolism and perform an effective infection. This phenomenon has been observed in several animal and plant pathosystems. Despite the relevance of this mechanism in pathogenesis, little is known about it in fungus-plant interactions. For that reason, we performed an in silico method to select plausible mimicry candidates for the Ustilago maydis-maize interaction. Our methodology used a tripartite sequence comparison between the parasite, the host, and nonparasitic organisms' genomes. Furthermore, we used RNA sequencing information to identify gene coexpression, and we determined subcellular localization to detect potential cases of colocalization in the imitator-imitated pairs. With these approximations, we found a putative extracellular formin in $U$. maydis with the potential to rearrange the host cell cytoskeleton. In parallel, we detected at least two maize genes involved in the cytoskeleton rearrangement differentially expressed under $U$. maydis infection; thus, this find increases the expectation for the potential mimicry role of the fungal protein. The use of several sources of data led us to develop a strict and replicable in silico methodology to detect molecular mimicry in pathosystems with enough information available. Furthermore, this is the first time that a genomewide search has been performed to detect molecular mimicry in a $U$. maydis-maize system. Additionally, to allow the reproducibility of this experiment and the use of this pipeline, we created a Web server called Molecular Mimicry Finder.
\end{abstract}

Keywords: coexpression, colocalization, formin, in silico method, molecular mimicry, Molecular Mimicry Finder, Ustilago maydis, Zea mays

${ }^{\dagger}$ Corresponding author: V. Armijos-Jaramillo; vinicio.armijos@udla.edu.ec

Funding: This research was supported by intramural funds of the Universidad de Las Américas-Quito (project BIO.VAJ.19.07).

*The $e$-Xtra logo stands for "electronic extra" and indicates that supplementary materials and supplementary files are published online.

The author(s) declare no conflict of interest.

(c) (1) (s) $\odot$ Copyright $\odot 2021$ The Author(s). This is an open access article distributed under the CC BY-NC-ND 4.0 International license.
Parasitic organisms have the ability to manipulate the host for their benefit. To achieve this, some microbial pathogens have evolved the capacity to mimic characteristics of their host. For example, the conidia of the fungus Monilinia vaccinii-corymbosi mimic the germinating pollen of the blueberry, thus enabling the fungus to gain access to the plant ovary (McArt et al. 2016; Ngugi and Scherm 2006). Similarly, the rice false smut fungus mimics successful fertilization (producing false smut balls). The imitation of the fertilized ovary allows for a continuous supply of nutrients (provided by the plant) for the fungus to survive (Song et al. 2016).

Extrapolating from morphological mimicry, the ability of one molecule to imitate another in function or shape is called molecular mimicry. The term was used for the first time by Damian (1964) to refer to the sharing of antigenic determinants between animal pathogens and hosts. Since then, several additional examples of molecular mimicry have been reported. For example, the human poxviruses and herpesviruses acquired the gene $\mathrm{Bcl}-2$ by horizontal gene transfer (HGT) from their hosts and, consequently, the viral protein $\mathrm{v}$-Bcl-2 mimics the function of the native protein and interferes with the normal apoptotic process of the host cell, benefiting the replication of the viral molecules (Elde and Malik 2009; Gangappa et al. 2002).

Bacteria, including Salmonella enterica and Escherichia coli, have an effector called IpgB2 that mimics the host Rho-GTPase enzyme and manipulates the cytoskeletal dynamics to increase the pathogen's fitness (Alto and Dixon 2008; Alto et al. 2006; Klink et al. 2010). Even in some autoimmune diseases such as Guillain-Barré syndrome, it has been proven that molecular mimicry between self and microbial components of Campylobacter jejuni is able to produce the disease (Shahrizaila and Yuki 2011). According to Loshaj-Shala et al. (2015), heat shock protein chaperones are suitable candidates for triggering GuillainBarré syndrome through molecular mimicry mechanisms.

One of the most studied cases of molecular mimicry involves the CLAVATA3 (CLV3)/endosperm surrounding region (ESR) (CLE) peptides of plants and phytopathogen nematodes. CLE proteins are involved in cell-fate determination in shoot and root apical meristems of Arabidopsis (Clark et al. 1995). Surprisingly, a gene encoding a CLE-like protein was found in the genome of the plant-pathogenic nematode Heterodera glycines (HgCLE proteins) (Olsen and Skriver 2003). Subsequently, it has been shown that nematode CLEs can cause root and shoot meristem termination and can complement Arabidopsis CLE mutants. Also, it has been shown that the continuous secretion of $\mathrm{HgCLE}$ proteins may be required to maintain the feeding cell in a dedifferentiated state (Wang et al. 2010, 2011; Wang et al. 
2005). These CLE proteins have been identified in several cyst nematodes, including potato cyst nematode, and there is evidence that they have evolved to utilize their secreted CLE effectors to activate multiple CLE signaling pathways through different host receptors to promote successful infection (Chen et al. 2015; Guo et al. 2011). Moreover, analyses of plantparasitic nematodes have revealed that CLE sequences are able to functionally complement the activity of their counterparts in plants (Mitchum et al. 2012).

Little is known about the effect of molecular mimicry in fungi as a pathogenic strategy. Nevertheless, a candidate effector from Melampsora larici-populina called chloroplast-targeted protein 1 (CTP1) carries a transit peptide that imitates the plant-specific sorting signals. The evidence shows that CTP1 uses the peptide to traffic within plant cell organelles, perhaps to manipulate the host (Petre et al. 2016). We can see another example of mimetic molecules in Fusarium oxysporum. This fungus has a functional homolog of plant peptide rapid alkalinization factor (RALF).
The pathogen apparently uses the peptide to suppress plant immunity and increase its infection potential (Masachis et al. 2016).

HGT or convergent evolution explain the molecular mimicry evolution. If an event of HGT results in molecular mimicry, sequence similarity is expected between the mimicking and imitated proteins. After the lateral transference event, strong selective pressure may maintain high sequence similarity; otherwise, the foreign gene will mutate to fit the host genome (amelioration process). In this case, the loss of the mimicry advantage is expected.

In contrast, when molecular mimicry is the result of convergent evolution, high sequence similarity is not expected and, to the contrary, structural similarities (tertiary conformation) are awaited. The events of convergent evolution may evolve via random mutations and natural selection (Ludin et al. 2011; Doxey and McConkey 2013; Drayman et al. 2013). Nevertheless, Hall (1994) suggests the concept of "consequential mimicry" to

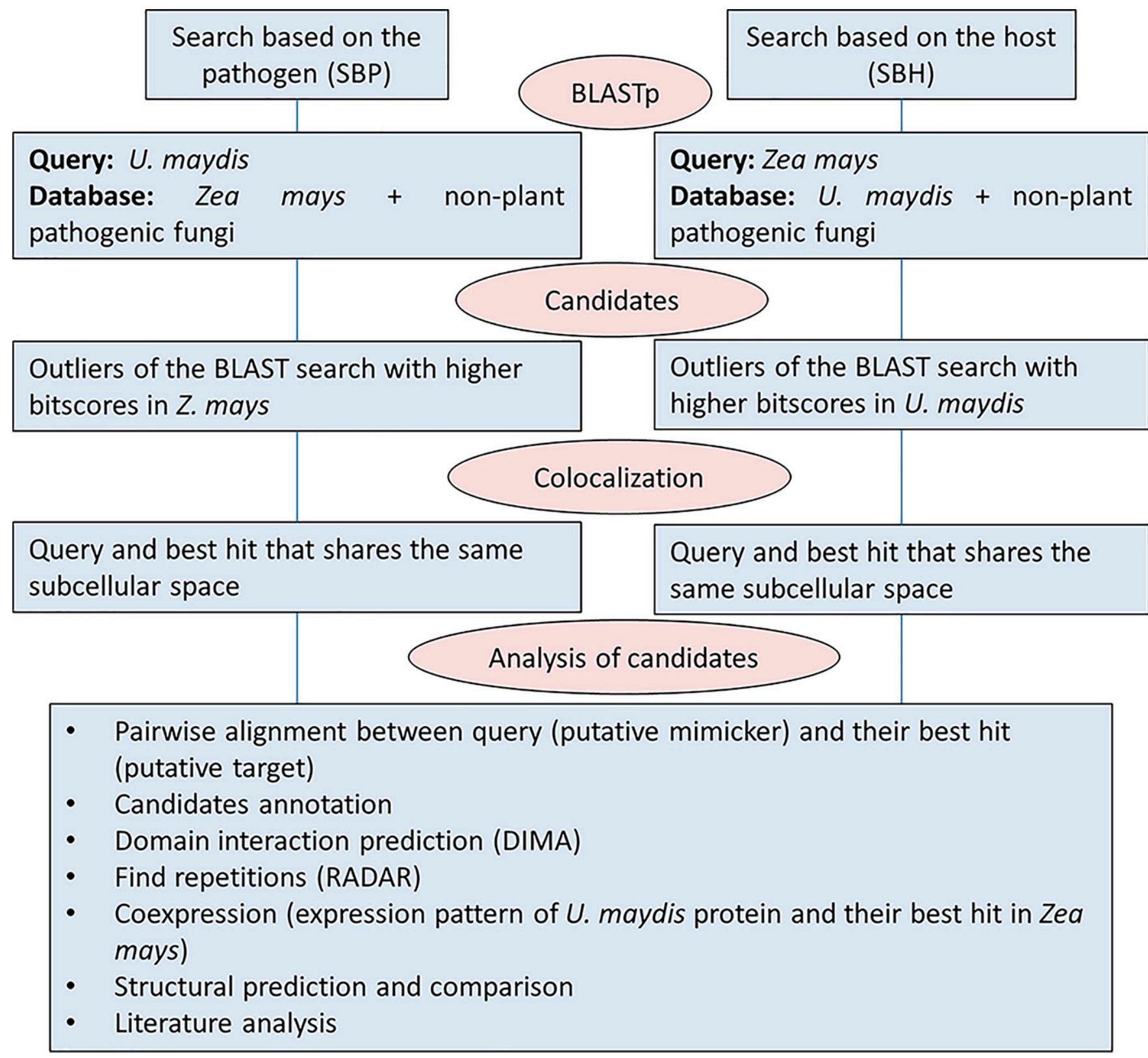

Fig. 1. General pipeline of the sequence similarity methods used to detect molecular mimicry candidates in Ustilago maydis. 
include the cases of phylogenetically preserved molecules. In this case, the molecular mimicry is the consequence of the conservation of sequences in a family of proteins that is used by the pathogen to imitate their host target. This scenario does not imply adaptation behind the molecular mimicry event but there is no restriction for the adaptation either.

Most of the verified examples of molecular mimicry to date are the result of functional experiments in the laboratory (Alto et al. 2006; Christensen and Kolomiets 2011; Wang et al. 2011). However, computational methods also have been developed to detect potential cases of molecular mimicry through the analysis of genome sequences. The first method was created to detect molecular mimicry in eukaryotic endoparasites of humans (Ludin et al. 2011) and the second one was developed to detect cases in human pathogenic bacteria (Doxey and McConkey 2013). Both methods are based on the detection of sequence similarity among host and parasite proteins. More recently, a similar strategy was used to find mimicry candidates in fish endoparasites (Hebert et al. 2015). Another in silico method was crafted in order to detect structural mimicry in several kinds of human parasites (Drayman et al. 2013). This method uses the structures deposited in the protein data bank (PDB) to compare microbe surface proteins with host ligands. This method has the advantage of exploring the molecular mimicry performed by convergent evolution but is limited by the number of structures deposited in the PDB.

Ustilago maydis is a basidiomycete smut fungus able to cause prominent symptoms in the aerial parts of its host (Zea mays) (Brefort et al. 2009). This pathogen induces the formation of local tumors a few days postinfection, a differential behavior among the biotrophic fungi. This fungus has been used as a model for the study of organisms with the same lifestyle (Lanver et al. 2017). Biotrophic fungi establish an intimate relationship with their host to keep themselves alive, and they reprogram the metabolism in order to sustain the feeding source. Specifically, $U$. maydis infects the plant through intracellular hyphae and produces an interaction zone to exchange molecules with plant cells (Djamei and Kahmann 2012). Bearing in mind this close interaction between parasite and host, the idea of molecular mimicry as a strategy to manipulate the host metabolism seems plausible for $U$. maydis and for the rest of biotrophic fungi.

Using the genetic information available for U. maydis, we developed a pipeline to detect molecular mimicry candidates using sequence similarity, colocalization, and coexpression patterns of genes and proteins. The lack of information surrounding molecular mimicry in plant-pathogenic fungi led us to develop an in silico approach to detect its presence in biotrophic fungi. The overall pipeline of the sequence similarity method is summarized in Figure 1. To the best of our knowledge, this is the first time that this kind of approximation has been applied in a biotrophic model such as $U$. maydis.

\section{RESULTS}

Identification of mimicry candidates by sequence similarity.

Molecular mimicry candidates were identified based on the sequence similarity methods of Ludin et al. (2011) and Doxey and McConkey (2013). In these studies, the authors compare the proteins of pathogens with those of the hosts and, as a control, proteins of nonpathogenic species closely related to the pathogen. It is expected that mimicry candidates have more sequence similarity to the proteins of their host than to the control species. The result is a pair of candidate proteins that represent the mimicker protein (encoded in the pathogen's genome) and the imitated protein (encoded in the host's genome). To perform this analysis, we retrieved the complete proteomes of 10 nonplant-pathogenic basidiomycetes species (controls) (Supplementary Table S1), U. maydis, and the proteome of Z. mays.

A BLAST search was performed using the proteins of $U$. maydis (search based on the pathogen [SBP]) as a query versus a database comprising proteins from nonplant-pathogenic fungi and the complete proteome of $Z$. mays. We selected as mimicry candidates the $U$. maydis proteins with outlier values of the bit score difference between the best hit in maize and the best hit in nonplant-pathogenic fungi (see Materials and Methods). Only three proteins obtained higher bit score values in maize than fungi but any that exceeded the fixed threshold were considered outliers (Fig. 2A).

A second BLAST search was performed using the proteins of Z. mays (search based on the host $[\mathrm{SBH}]$ ) as a query versus a database comprised of the proteomes of $U$. maydis and nonplant-pathogenic fungi. We selected proteins with outlier values of the bit score difference between the best hit in U. maydis and the best hit in nonplant-pathogenic fungi (see Material and Methods). We obtained 111 maize proteins in this search (Fig. 2B).

Molecules that mimic proteins of the host are frequently colocalized to the same subcellular space as the imitated protein
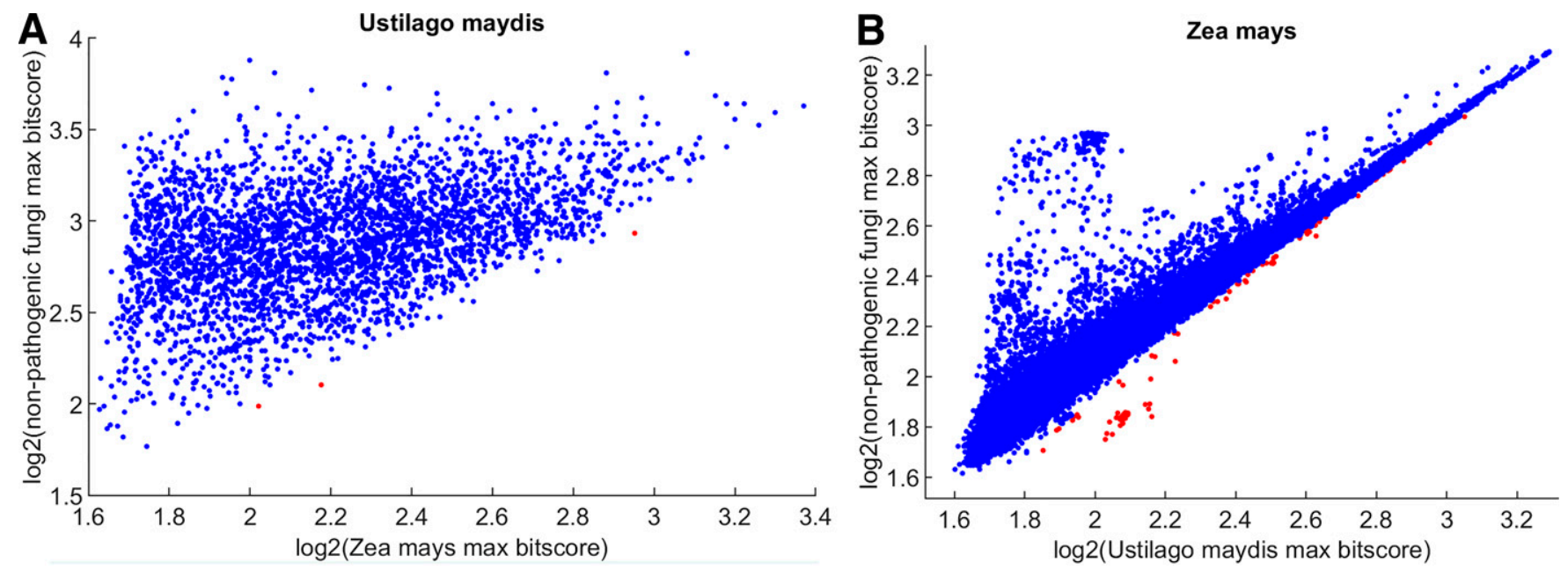

Fig. 2. Scatter plots of the best BLAST hits for the whole proteome of Ustilago maydis (pathogen) and Zea mays (host) versus nonplant-pathogenic fungi (control). A, Red dots represent $U$. maydis proteins with higher bit scores to Z. mays proteins than to nonplant-pathogenic fungi. B, Red dots represent Z. mays proteins with higher bit scores to $U$. maydis than nonplant-pathogenic fungi. 
(Alto et al. 2006; Drayman et al. 2013; Gangappa et al. 2002). For example, the proteins SopE and SopE2 of the human enteric pathogen Salmonella are secreted through the type III secretion system to the host cytoplasm. Inside the host, these proteins mimic guanine nucleotide exchange factors (GEFs) that activate Rac1 and Cdc4 (GTPases), leading to the rearrangement of the actin cytoskeleton and subsequent bacterial internalization (Galán and Zhou 2000). These events require that the mimicking proteins (SopE and SopE2) and the imitated proteins (human GEFs) share the same subcellular space-in this case, the host cytoplasm. For that reason, we predicted the subcellular localization for each pair of candidates (U. maydis and Z. mays proteins) obtained in the BLAST searches described above. We deduced that the subcellular localization of the pair is congruent only if the $U$. maydis or Z. mays protein is secreted and its pair in the fungus is a transmembrane protein. A schematic view of the probable interacting proteins between $U$. maydis and Z. mays is shown in Figure 3. We obtained only two pairs of candidates after applying these criteria. Another 12 pairs with transmembrane annotation in fungus and maize were found; however, we rationalized that these are not able to share the same subcellular space because the fungal cell wall separates them. In that sense, these are less plausible mimicry candidates (Supplementary Table S2).

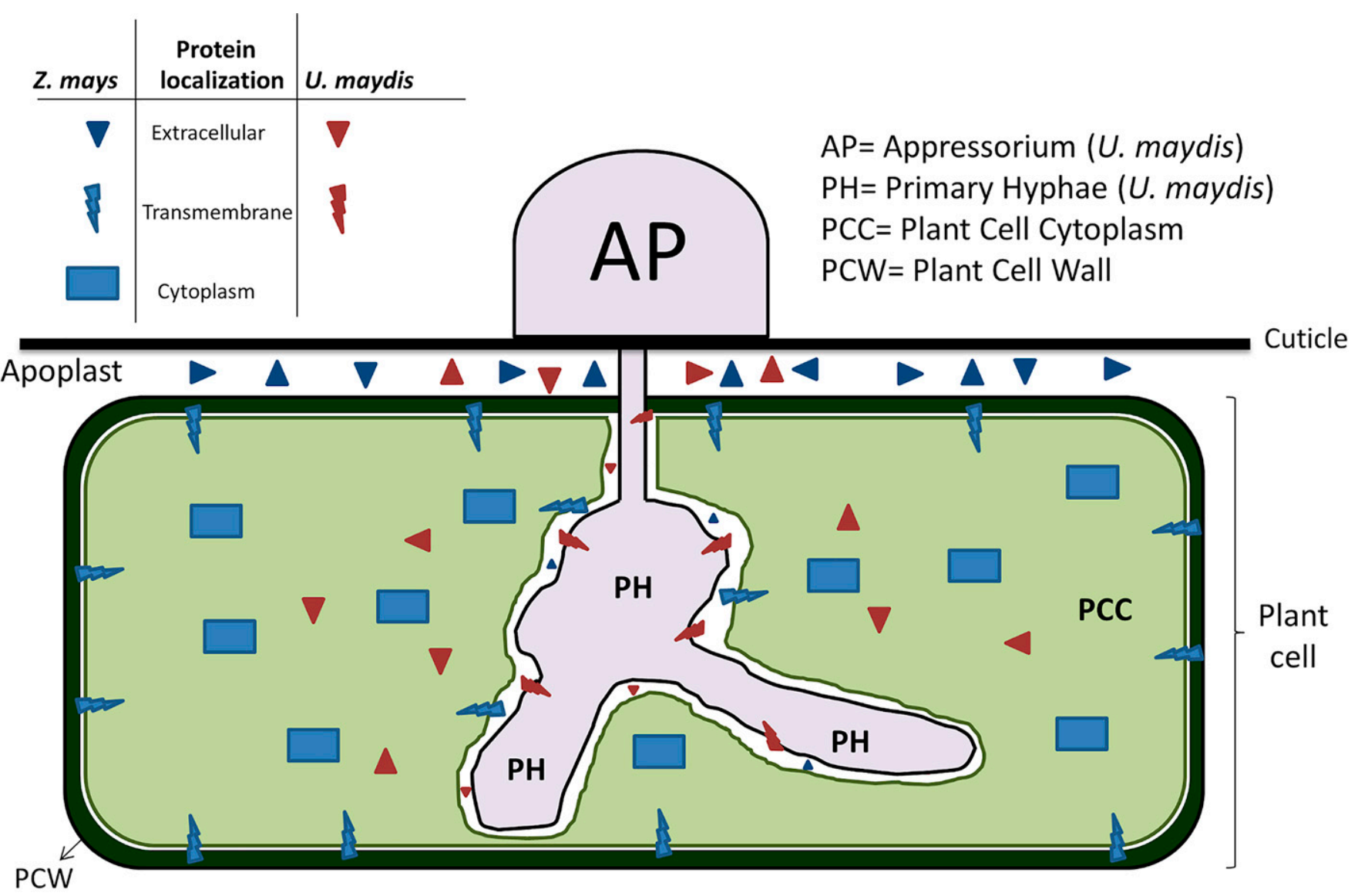

Fig. 3. Schematic view of the subcellular localization of mimicry candidates and imitated Zea mays proteins. Secreted Ustilago maydis proteins could be in the apoplast or plant cell cytoplasm; consequently, these proteins could imitate plant proteins inside or outside the plant cell. Transmembrane proteins of $U$. maydis could interact with the extracellular proteins of $Z$. mays but not with the transmembrane proteins of the plant cell because the fungal cell wall (not represented in the figure) separates the space between them.

Table 1. Ustilago maydis mimicry candidates colocalized in the same subcellular space of their putative imitated maize protein

\begin{tabular}{|c|c|c|c|c|c|c|}
\hline \multicolumn{3}{|c|}{ Ustilago maydis } & \multicolumn{3}{|c|}{ Zea mays } & \multirow[b]{2}{*}{$\begin{array}{c}\text { Pairwise } \\
\text { similarity }(\%) \\
\end{array}$} \\
\hline Locus number & $\begin{array}{l}\text { Subcellular } \\
\text { localization }\end{array}$ & $\begin{array}{c}\text { Interpro } \\
\text { domain IDs }\end{array}$ & Locus number & $\begin{array}{l}\text { Subcellular } \\
\text { localization }\end{array}$ & $\begin{array}{c}\text { Interpro } \\
\text { domain IDs }\end{array}$ & \\
\hline A0A0D1CAH9_USTMA & Extracellular & $\begin{array}{l}\text { IPR040693, } \\
\text { IPR009448 }\end{array}$ & A0A1D6HNS8_MAIZE & Extracellular & $\begin{array}{l}\text { IPR040693, } \\
\text { IPR040694, } \\
\text { IPR040692, } \\
\text { IPR040525, } \\
\text { IPR009448, } \\
\text { IPR040497 }\end{array}$ & 42.5 \\
\hline A0A0D1CXZ5_USTMA & Extracellular & $\begin{array}{l}\text { IPR010473, } \\
\text { IPR010472, } \\
\text { IPR014768, } \\
\text { IPR015425, } \\
\text { IPR016024 }\end{array}$ & A0A1D6F098_MAIZE & Cytoplasm & $\begin{array}{l}\text { IPR029023, } \\
\text { IPR014020, } \\
\text { IPR015425 }\end{array}$ & 33.9 \\
\hline
\end{tabular}


Domains detected in candidates were annotated with InterProScan (Table 1) and PHI-base was used in $U$. maydis proteins to determine similarities with previous fungal proteins involved in pathogenicity. In PHI-base, we found only one relevant result for A0A0D1CAH9_USTMA that is similar to the KRE5 of Colletotrichum graminicola. In one pair of candidates (A0A0D1CXZ5_USTMA and A0A1D6F098_MAIZE), we found a proline-rich region. These regions were not associated with any specific domain predicted by InterPro.

We used the Domain Interactions Map (DIMA) tool (Luo et al. 2011) to determine whether the mimicry candidates interact with their imitated Z. mays proteins or other proteins. The prediction of interacting domains can also give us clues regarding the putative target proteins of the mimicking candidate. At least one candidate (A0A0D1CXZ5_USTMA) could interact with the same domain (to form dimers) (Supplementary Table S3). It is also possible that such interacting proteins could form host-pathogen heterodimers, as reported by Chan et al. (2009).

The phylogenetic distribution of the $U$. maydis candidates and the Z. mays ones were investigated to determine whether they are the product of HGT. We performed BLASTP searches versus the NR database of NCBI and then reconstructed a phylogenetic tree. Both candidates (in $U$. maydis and maize) have a phylogenetic distribution congruent with the species tree; thus, it is unlikely that these genes evolved by HGT.

\section{Coexpression of mimicry candidates and maize genes.}

The mimicking and the imitated genes could share a similar expression pattern throughout the infection process. This behavior could increase the opportunities of the pathogen's protein to imitate their counterpart because it is localized in the correct place (colocalized) and at the correct time (coexpressed). Evidently, this is not the only option for performing the molecular mimicry but the coincidence of expression patterns could reflect a fine tuning between two phylogenetically unrelated organisms. The expression of each pair of candidates was explored on infected maize leaves with $U$. maydis at $0.5,2,4,6$, 8 , and 12 days postinfection (dpi); in addition, axenic culture of $U$. maydis and uninfected (monk) tissue for Z. mays were used as controls.

The normalized read count for $U$. maydis was obtained from the RNA sequencing (RNA-seq) data from Lanver et al. (2018) and the data for $Z$. mays were calculated from the raw data of the same work. The differential expression calculus for Z. mays genes is shown in Supplementary File S1. The expression profile of the candidates (in U. maydis and Z. mays) can be observed in Figure 4. Differential expression was considered when $P$ values (adjusted for multiple comparisons with Tukey's honestly significant difference) were lower than 0.01 in comparison with the controls.

\section{Structural comparison of candidates.}

We reconstructed the candidates' three-dimensional (3D) structure to observe the similarities at this level in $U$. maydis proteins compared with the maize ones. In the two pairs of candidates, we found that Phyre 2 used the same templates to model $U$. maydis and Z. mays proteins. However, several differences in the $3 \mathrm{D}$ reconstruction were observed. For example, in

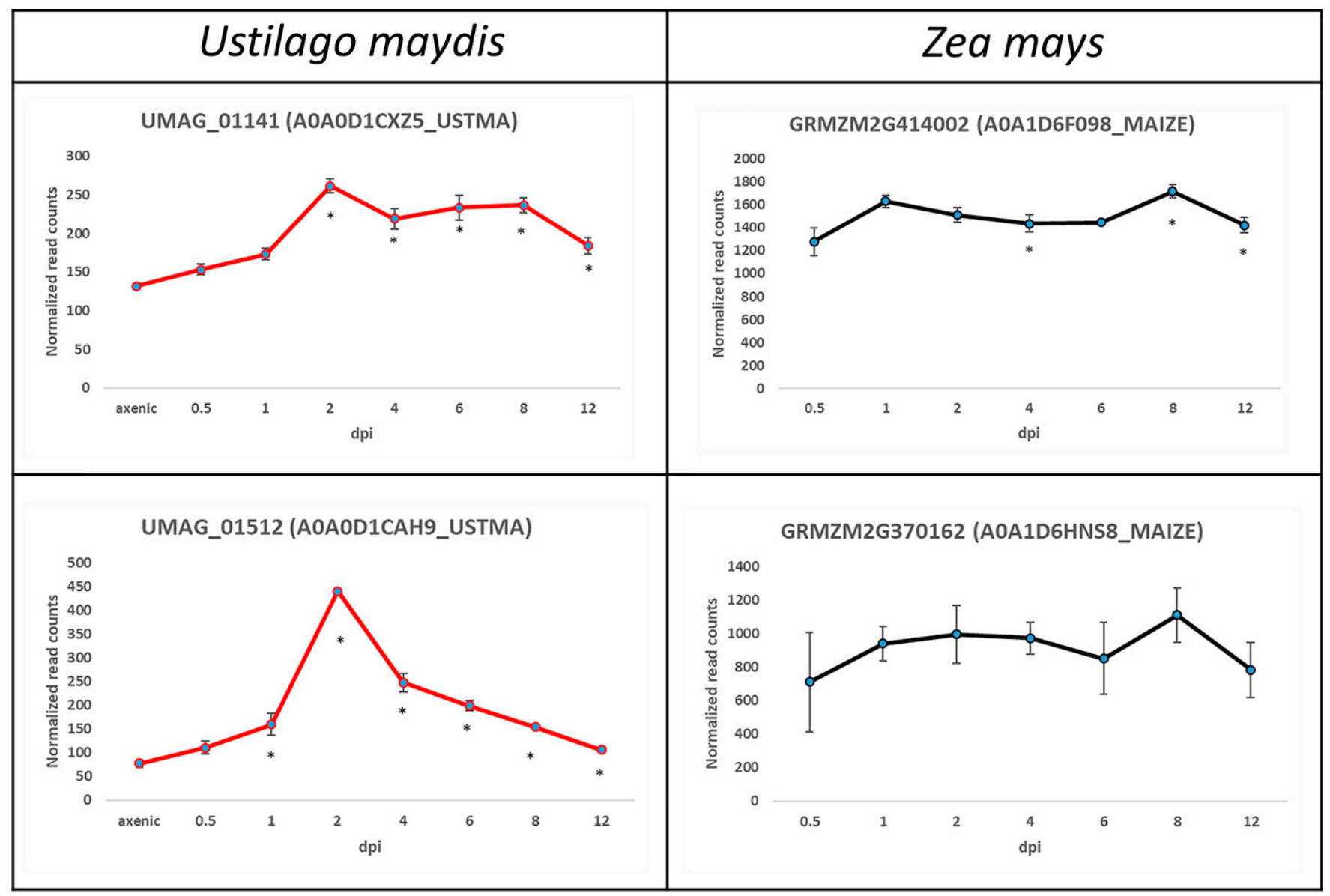

Fig. 4. Expression profile (mean) of mimicry-imitated candidate pairs (Ustilago maydis and Zea mays). Expression was measured at 0.5, 2, 4, 6, 8, and 12 days postinfection (dpi). Axenic culture expression of $U$. maydis is also included. Vertical bars at each point represent standard deviation. Asterisks above graph points show differential expression $(P$ adjusted $>0.01)$ compared with axenic culture in $U$. maydis and with the same time point with plants that were mock infected with water. 
A0A0D1CXZ5_USTMA, 87\% of the residues were modeled by homology and $13 \%$ ab initio (unreliable) versus the $55 \%$ of ab initio residues modeled for A0A1D6F098_MAIZE. Superimposition of these structures gave a root-mean-square deviation (RMSD) of atomic positions of 5.51. Only 7\% of the residues were reconstructed ab initio for A0A0D1CAH9_USTMA and 13\% for A0A1D6HNS8_MAIZE. Phyre2 calculated an RMSD of 1.75. The superimposition of the two pairs of structures is observed in Figure 5.

Detection of molecular mimicry examples using the pipeline.

We used the pipeline described in this work to test its power to detect well-established examples of mimetic proteins. The first case used was the peptide RALF (A0A0J9VUZ1), detected in F. oxysporum (Masachis et al. 2016). We were able to detect this molecule using SBP and SBH. With the SBH, we detected as putative imitated proteins seven RALF peptides annotated in the proteome of Solanum lycopersicum (A0A3Q7EGN8, A0A3Q7EN81, A0A3Q7EVK7, A0A3Q7IB50, A0A3Q7J1D9, A0A3Q7J1I1, and A0A3Q7JYR0). With the SBP, only one proteins of $S$. lycopersicum were detected (A0A3Q7EGN8). The complete list of candidates obtained from the analysis can be observed in the Supplementary File S2.

We used the pipeline to detect the CLE-like peptides of $H$. glycines (Hg-CLE), mimicry molecules of the CLE in plants (Wang et al. 2005). We detected CLE1 (Q9BN21) and CLE2 (Q86RQ1) of $H$. glycines with the SBP and SBH as mimicry candidates. Additionally, we detected six Arabidopsis CLE proteins (Q3EDH8, Q3ECD6, Q8S8N2, O49519, Q8S8N3, and A0A1P8B327) as putative imitated molecules. The complete list of candidates detected can be obtained from Supplementary File S2.

\section{DISCUSSION}

In this work, we used an in silico pipeline to detect the most plausible proteins in $U$. maydis that are able to imitate their counterparts in Z. mays. To achieve this goal, we applied two approaches which are based on sequence similarity between the mimicking protein and the imitated protein and that were originally developed for detecting mimicry in human pathogens
(Doxey and McConkey 2013; Ludin et al. 2011). Additionally, we rationalized the need of a mimetic protein to be in the same subcellular space as the imitated molecule to perform their function for the pathogen. In that sense, we filtered the candidates obtained in the sequence similarity step by their putative subcellular space location. We considered the candidates with unshared subcellular space with their counterparts in Z. mays as potential cases of consequential mimicry with or without an adaptive history related to coevolution with the host. For pairs of proteins ( $U$. maydis and Z. mays) with sequence similarity and subcellular location congruence, we analyzed the expression patterns under infection conditions to explore the coexpression possibility. Considering all of these criteria, we found at least one candidate in $U$. maydis with the potential to imitate specific Z. mays proteins in shape or function.

\section{$U$. maydis proteins with the potential to imitate $Z$. mays sequences.}

A first candidate (A0A0D1CAH9_USTMA) was annotated as UDP-glucose: glycoprotein glucosyltransferase (UGGT), a glycoprotein able to bind, and glycosylates unfolded proteins (UDP-glycoprotein; PTHR11226). These kinds of proteins play an important role in quality control inside the endoplasmic reticulum (ER). In Arabidopsis thaliana, mutants deficient in UGGT were more sensitive to pathogen attack and showed difficulties in establishing systemic acquired resistance (Blanco-Herrera et al. 2015). Despite the fact that both proteins (A0A0D1CAH9_USTMA and A0A1D6HNS8_MAIZE) are predicted with a signal peptide, the annotation means that it is highly unlikely that these molecules migrate out of the cell. This is because the role of UGGTs is strongly associated with the ER. For that reason, it is difficult to imagine a UGGT from fungi imitating a plant protein with the same function. The signal peptide observed in these molecules might be associated more with ER than extracellular destination. These kinds of ambiguous targeting signals have been observed in other organisms (Kunze and Berger 2015). In any case, the presence of a signal peptide in this group of proteins is common. From a random sample of 60 proteins annotated as UGGT in InterPro, 28 were predicted with a signal peptide (data not shown) using SignalP software.

The expression of codifying genes of A0A0D1CAH9_USTMA and A0A1D6HNS8_MAIZE did not show similar
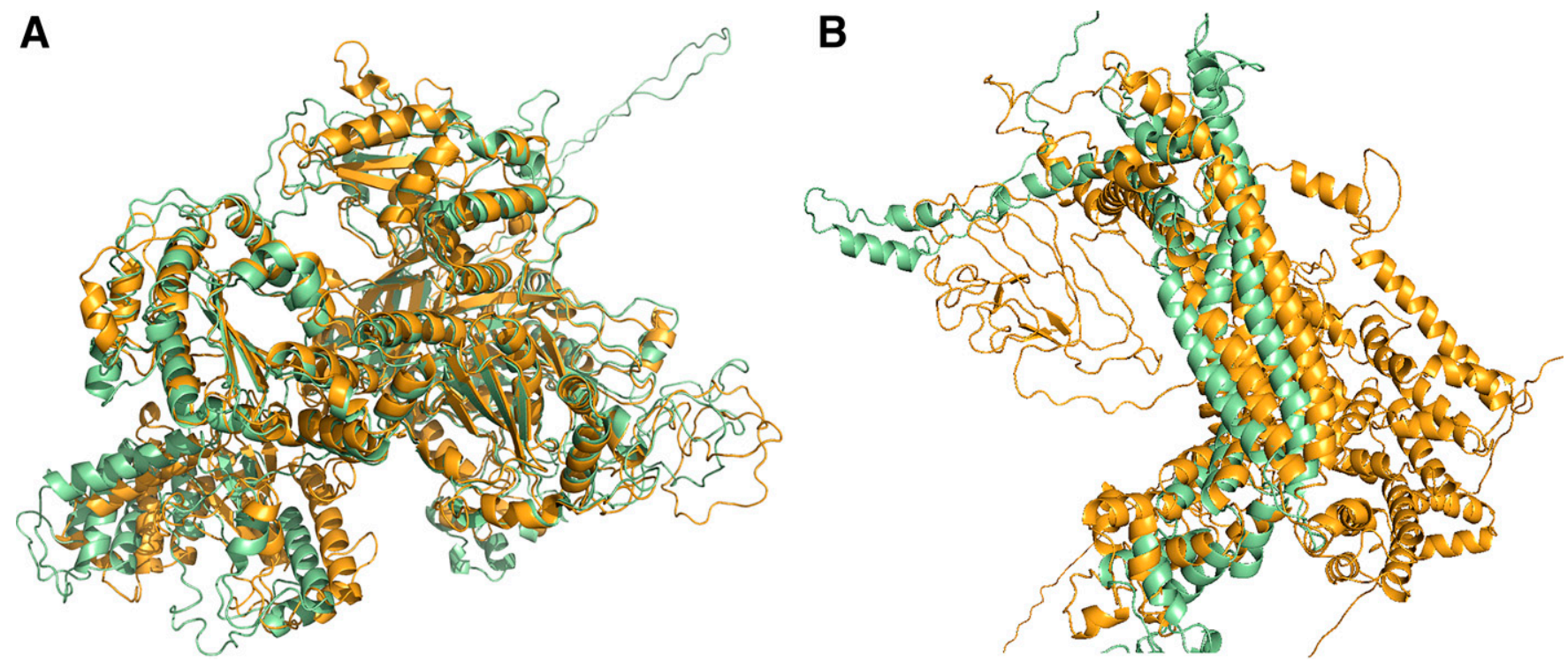

Fig. 5. Three-dimensional superimposition of mimicry (Ustilago maydis) and imitated (maize) candidates. A, Pair A0A0D1CAH9_USTMA and A0A1D6HNS8_MAIZE. B, Pair A0A0D1CXZ5_USTMA and A0A1D6F098_MAIZE. U. maydis proteins are represented in bright orange and maize proteins in pale green. 
patterns over time. Moreover, the expression of the maize gene seemed unaffected (no difference with the mock plants was detected) by the infection of $U$. maydis. However, the structural similarities between $U$. maydis and maize proteins are remarkable (RMSD of 1.75). This level of similarity between proteins can be easily confounded as an example of mimicry. To avoid confusion, it is necessary to evaluate several sources of information before making any conclusions. Without the evidence obtained with the pipeline applied in this article (sequence and structural similarity, expression pattern, subcellular localization, and annotation), more than one candidate could be mistaken as a mimicry candidate. For the case of A0A0D1CAH9_USTMA, the predicted subcellular localization, sequence, and structural similarity support the mimicry scenario but the coexpression pattern and, especially, the annotation as UGGT make this hypothesis improbable.

A0A0D1CXZ5_USTMA and A0A1D6F098_MAIZE were identified as formins. These proteins are involved in the polymerization (nucleation) of actin in the cytoskeleton (also the actin filament elongation). Additionally, depolymerization, severing, and bundling actin and microtubule binding have been associated with these proteins (Breitsprecher and Goode 2013). The ability of formins to interact with other proteins and perform a specific function depends on domain composition. In the case of A0A0D1CXZ5_USTMA and A0A1D6F098_MAIZE, both share the $\mathrm{FH} 2$ domain (IPR015425) in the C-terminal region and a proline-rich region before $\mathrm{FH} 2$. This proline-rich region is characteristic of the FH1 domain (Takeya and Sumimoto 2003); however, InterPro cannot determine it as such. The difference between maize and $U$. maydis proteins are observed in the N-terminal region. A0A1D6F098_MAIZE has a tensin phosphatase domain (IPR029023) and a tensin C2-domain (IPR014020). Tensin-type phosphatase domains are well characterized in animals, especially in the PTEN tumor suppressor protein (Leslie and Downes 2002), but are not so well described in plants. However, the Prot-tyrosine_phosphatase-like domain (IPR029021) was also annotated in the same position as IPR029023. In plants, proteins with these domains have been related to the regulation of the mitogen-activated protein kinase signaling cascade (Shankar et al. 2015). None of these domains is predicted in the N-terminal region for A0A0D1CXZ5_USTMA. However, in their place, a signal peptide, FH3 domain (IPR010472), and a GTPase-binding domain (IPR010473) were observed. Point mutations in mammalian cells showed that FH3 seems necessary in order to locate the formins in the correct cellular location (Kato et al. 2001), and the GTPase-binding domain is required for binding to Rho small GTPases, which leads the cytoskeletal rearrangements by formin activation (Kühn and Geyer 2014).

The differences observed in the domain composition between A0A0D1CXZ5_USTMA and A0A1D6F098_MAIZE are also reflected in the structural superimposition (Fig. 5B). The direction of $\alpha$ helixes seems different in both proteins (RMSD $=$ 5.51). These variances could be lower or higher but, with the current model obtained for A0A1D6F098_MAIZE (55\% of sites predicted ab initio), it is difficult to ensure a lack of structural similarity between these proteins.

In the codifying genes of A0A0D1CXZ5_USTMA and A0A1D6F098_MAIZE, a differential expression under infection conditions was observed (Fig. 3; Supplementary File S1). In the gene GRMZM2G414002 that codifies A0A1D6F098_MAIZE, a lightly but significant repression was observed from 4 to 12 dpi in comparison with the uninfected plants (mock). Additionally, we observed a significant overexpression of GRMZM2G117603 from $12 \mathrm{~h}$ postinfection to $12 \mathrm{dpi}$. GRMZM2G117603 codifies the actin-depolymerization factor 1 (ADF1), a protein that binds to actin monomers to disassemble the cytoskeleton (Tholl et al. 2011). Direct interaction between formins and ADF1 have not been observed; however, both are involved in the constant modification of cytoskeleton.

The putative extracellular localization of the formin A0A0D1CXZ5_USTMA raises an intriguing question: is this molecule manipulating the host cytoskeleton? A similar phenomenon has been observed in bacteria. Pathogenic animal bacteria use extracellular proteins (effectors) to manipulate the host's cytoskeleton to facilitate cellular invasion (Alto and Dixon 2008; Alto et al. 2006; Klink et al. 2010). These effectors alter the endothelial barriers and lead to the local rearrangement of the cytoskeleton by the manipulation of the Rho GTPases cascade. With these modifications, bacteria are able to mediate their entry into the cell and avoid phagocytosis (Popoff 2014). Coincidently, for the formin A0A0D1CXZ5_USTMA, a GTPase-binding domain was identified. This domain interacts with Rho GTPases, and the domains FH1, FH2, and FH3 interact with actin. It is clear that this putative formin could play a role in the cytoskeleton rearrangement.

The remaining question is whether A0A0D1CXZ5_USTMA works in the plant or in the fungal cell. In order to shed light on this question, we studied the domain conformation of the formin paralogue A0A0D1CLP7_USTMA identified in the U. maydis proteome (in agreement with the EnsemblFungi website). We were able to annotate the domains $\mathrm{FH} 2$ and $\mathrm{FH} 3$ and also the aGTPase-binding domain but not a signal peptide. In the absence of experimental evidence, the bioinformatic predictions dictate the presence of at least two formins in $U$. maydis, one for the fungal cell and the other potentially exported. The extracellular formin has all of the elements needed to manipulate the host cell's cytoskeleton, mimicking the function of the native plant formins.

\section{A pipeline to detect molecular mimicry candidates.}

The majority of the methods developed to search for molecular mimicry focus on epitopes. In silico sequence-based approaches such as those of Kubrycht and Novotná (2014), Ansari Qeshmi et al. (2016), Bhardwaj et al. (2018), Múnera et al. (2020), and Portnyagina et al. (2018), the focus is on detecting short sequence similarities between the mimicker and the imitated molecule. Studies such as those of Ludin et al. (2011), Doxey and McConkey (2013), and Hebert et al. (2015) evaluate entire sequence similarities between host and pathogen proteins, while Drayman et al. (2013) analyzed structural similarities. Following the approximation of these articles, we developed a preliminary pipeline using Colletotrichum graminicola-maize as a model system (Armijos-Jaramillo 2014). Several candidates with the potential to imitate maize proteins were detected in that study and we decided to improve the methodology by including structural comparisons to consider other ways to perform mimicry by the pathogens. Also, we decided to work on the $U$. maydis $-Z$. mays pathosystem in order to gain information so as to annotate the pathogen genomic.

HGT and convergent evolution are two mechanisms by which molecular mimicry could have evolved (Ludin et al. 2011; Doxey and McConkey 2013; Drayman et al. 2013; Stebbins and Galán 2001). In the case of convergent evolution, the two proteins involved (imitator and imitated) may have a low similarity at the sequence level, although they most likely have structural similarity. In the case of HGT, sequence similarity is expected (Drayman et al. 2013). These two options are incongruent with the results of Ludin et al. (2011), Doxey and McConkey (2013), and Hebert et al. (2015) because these authors found molecular mimicry candidates by sequence similarity but without evidence of HGT in most of the cases. This observation contradicts the expectation that molecular mimicry could have evolved by HGT. To detect candidates of molecular mimicry by sequence resemblance between the mimicker and the imitated protein, another evolutionary explanation is needed. The presence of 
conserved gene families in unrelated organisms and the presence of selective pressures (e.g., the need of the pathogen to infect the host) may explain these results. A similar evolutionary scenario was described by Hall (1994) with the use of the term "consequential mimicry". This term, in contrast to adaptative mimicry (produced by HGT or convergent evolution), is the result of sharing phylogenetically conserved genes in distantly related organisms. Consequential mimicry does not imply adaptation but, equally, does not exclude it. We propose that the method based on sequence similarity is able to detect cases of molecular mimicry that fit with the concept of consequential mimicry (with or without adaptation) or cases that are the result of HGT. The molecular mimicry cases produced by convergent evolution can be detected by structural analysis. For that reason, we worked with the predicted 3D models of the candidates to evaluate structural conformation similarities that allow imitation of the function of plant protein without a high level of primary sequence similarity. It is clear that the pipeline presented in this study is not able to detect by itself cases of structural mimicry, and at least a low level of similarity between pathogen and host proteins is needed.

In this study, we utilized subcellular localization as an additional criterion to identify mimicry candidates. We used the colocalization criterion to filter the list of BLAST search results. Two colocalization scenarios were proposed. First, the secreted proteins of $U$. maydis could imitate the extracellular or cytoplasmic proteins of the host (Kamoun 2007; Koeck et al. 2011; Rafiqi et al. 2013). Second, the extracellular proteins of maize could interact with the extracellular proteins of $U$. maydis. The usage of this criterion increases the chances of detecting real mimicry proteins because it is necessary to imitate the function of the host molecules in order to occupy the same subcellular space. The success of this condition is limited by the accuracy of the subcellular prediction methods. The colocalization rationale could be insufficient if the pathogen molecule arrives at the correct subcellular space out of time. To overcome this possible scenario, we decided to study the expression pattern of the candidates under infection conditions. With this information, we were able to establish whether a candidate affected the host during the infection time when the imitated genes were expressed. It is important to emphasize that the coexpression is not the only possible scenario for molecular mimicry. If the mimicker protein inhibits the expression of its target (as in a negative feedback system), the expression pattern expected in the plant's genes is the opposite of the pathogen's genes.

Additionally, using this pipeline, we were able to detect two well-documented mimicry examples: RALF peptides (A0A0J9VUZ1) of $F$. oxysporum (Masachis et al. 2016) and CLE of $H$. glycines (Wang et al. 2005). The $F$. oxysporum RALF peptide was detected with SBH and SBP with the same parameters described in the Materials and Methods section. However, only $H$. glycines CLE2 (Q86RQ1) could be detected in SBH using the interquartile range to detect candidates with the higher bit score difference with other BLAST hits. This result shows that relevant mimicry candidates can be detected with low bit score differences. For that reason, in the Web server "Molecular Mimicry Finder", we implemented the complete list of candidates with bit score difference $>0$ as results. Furthermore, the user can choose a factor for the interquartile range, in order to select only the candidates with the highest bit score differences. Then, the Web server provide this list of candidates with the subcellular localization annotation available in UniProt database. At the end of the process, the user receives four documents: a list of all results with bit score difference $>0$ for $\mathrm{SBH}$ and SBP, and a list of candidates with the highest bit score differences (defined by the interquartile range) with subcellular localization annotations, also for SBH and SBP. The results shared by SBH and SBP with the higher bit score difference should be consider the strongest candidates; however, it depends on the pathosystem, as was demonstrated in the detection of CLE peptides in $H$. glycines.

The pipeline described in this work was able to detect at least one protein in $U$. maydis with the characteristics to perform molecular mimicry in maize plants during infection. The strict criteria used for the search led us to detect such a low number of candidates. Several factors-for instance, an insufficient number of unrelated control species or the loss of gene families in unrelated control species-could mislead mimicry candidate identification, resulting in false positives. As mentioned before, the accuracy in the subcellular localization predictions can also mislead the candidate's identification, as was discussed for the candidate A0A0D1CAH9_USTMA. The scarce number of crystalized proteins of $U$. maydis and $Z$. mays also affects the method's capability to detect structural similarities in the molecular mimicry candidates. For all of these reasons, the application of the current pipeline is limited to pathosystems with certain information available. It needs at least the genomic information of pathogen-related species (without the same lifestyle), expression data under infection conditions, and, if possible, high-quality annotated proteomes for the host and the pathogen.

\section{Conclusions.}

In this study, we detected at least one protein in $U$. maydis with the potential to imitate and perhaps manipulate maize proteins. A0A0D1CXZ5_USTMA has domains that interact and rearrange the actin filaments of the cytoskeleton, plus a signal peptide to localize outside the fungal cell. Additionally, the codifying gene of this protein is overexpressed under maize infection conditions. In parallel, we detected at least two maize genes involved in cytoskeleton rearrangement with differential expression at the same time points. These observations highlight the importance of cellular cytoskeleton dynamics during the infection of $U$. maydis in maize and reinforce the relevance of A0A0D1CXZ5_USTMA.

In addition, we were able to create a strict and biologically congruent pipeline to detect candidates of molecular mimicry in pathosystems. Only with the first part of the pipeline (BLAST searches) were well-stablished cases of molecular mimicry detected, showing the power of the method to detect putative mimetic proteins. The usage of sequence and structural similarities led to the opportunity of detecting mimicry candidates with different evolutionary origins, and the use of expression and subcellular localization data allowed for enclosing candidates with biological relevance. We created a Web server called Molecular Mimicry Finder to allow the public to reproduce this pipeline (from the BLAST searches to subcellular localization annotation) with different pathosystems.

The study of molecular mimicry opens up a new perspective in our knowledge regarding plant-microbe interactions. The ability of fungi to mimic plant proteins involved in defense suggests the evolution of very specific tactics used to invade their host. This gives us the opportunity to identify new components of the plant's immune system that can lead to the development of new disease-control strategies. Likewise, the improvement of in silico molecular mimicry detection methods opens up an exciting field of research for molecular plant pathology.

\section{MATERIALS AND METHODS}

\section{Identification of molecular mimicry candidates by sequence similarity.}

The proteomes of $U$. maydis, Z. maydis and the nonplantpathogenic fungi (Supplementary Table S1) were downloaded from UniProt. 
For the first comparison, a BLASTP (Altschul et al. 1997) (e-value $=1 \mathrm{E}-5)$ search based on the pathogen (SBP) was performed with all proteins predicted in the genome of $U$. maydis 521/FGSC 9021 versus the database made of the nonplantpathogenic fungi proteomes (Supplementary Table S1) plus the maize proteome. For the second comparison, a BLASTP (e-value $=1 \mathrm{E}-5)$ search based on the host $(\mathrm{SBH})$ was performed with all of the proteins predicted in the genome of maize versus the database comprised of nonplant-pathogenic fungi and $U$. maydis 521/FGSC 9021.

From the BLAST results, we calculated in the SBP the bit score difference between the best hit in maize and the best hit in nonplant-pathogenic fungi for every $U$. maydis protein. For $\mathrm{SBH}$, the bit score difference between the best hit in $U$. maydis and the best hit in nonplant-pathogenic fungi was calculated for every maize protein. Then, we plotted these data in boxplots and took the outliers (higher than Q3 + interquartile range) as mimetic candidates' proteins. The results without BLAST results in nonplant-pathogenic fungi but with results in maize or $U$. maydis were also added to the candidates lists.

\section{Prediction of subcellular localization.}

SignalP 4.1 (Petersen et al. 2011), WolfPsort v0.2 (Horton et al. 2007), and TMHMM 2.0 (Krogh et al. 2001) were used to annotate the putative subcellular localization of the candidates. When more than two transmembrane sites were predicted in the protein, it was annotated as a transmembrane protein without taking into account the other annotations. When a signal peptide was predicted (by SignalP or WolfPsort) with two or fewer transmembrane sites, the proteins were predicted as extracellular. Finally, if the protein was predicted to contain one or two transmembrane sites and without signal peptide, this was annotated with the WolfPsort category.

The mimicry candidates and their imitated maize proteins were filtered based on their predicted subcellular localizations. Two combinations of subcellular localization were considered. Extracellular proteins of $U$. maydis may colocalize with any maize protein and the transmembrane proteins of $U$. maydis may be able to interact with the extracellular proteins of maize. The rest of the combinations would be highly unlikely to occur. The colocalized candidates were annotated with InterPro (Hunter et al. 2012), MEROPS batch BLAST analysis (Rawlings et al. 2016), and BLAST analysis to PHI-base (Urban et al. 2017).

\section{Domain interaction prediction and phylogenetic analysis.}

The pairwise alignments ( $U$. maydis-maize pairs) were performed with MAFFT (Katoh and Standley 2013). The search for tandem repeats was performed with RADAR (Heger and Holm 2000). The proteins were considered repetitive when RADAR detected more than four repeats with a total score greater than 100. Protein domain interactions were identified candidate by candidate with the DIMA (Luo et al. 2011).

To reconstruct the phylogenies of the mimicry candidates, BLASTP searches versus the NR database of NCBI were performed with each $U$. maydis and maize candidate. When more than one kingdom was observed in the BLAST hits of a protein (e.g., fungus, animal, bacterium, and plant), a phylogenetic tree was reconstructed using PhyML (Guindon et al. 2010) to evaluate the possibility of HGT.

\section{Gene expression analysis.}

The RNA-seq experiments of Lanver et al. (2018) were retrieved from the Gene Expression Omnibus (GEO ID GSE103876) database and the normalized counts data were used for the differential expression analysis of the candidates. This experiment contains data of maize leaves infected with $U$. maydis with samples collected at $0.5,1,2,4,6,8,10$, and 12 dpi.
The differential expression of genes in $U$. maydis and maize were calculated with the package DESseq (Anders and Huber 2010) based on the negative binomial distribution of $R$ ( $R$ Development Core Team 2011).

\section{Structural prediction of candidates and superimposition.}

The 3D structures of the candidates were predicted by homology with the Phyre 2 server (intensive modeling mode) (Kelley et al. 2015). Given that the intensive mode restricts the length of the sequence to 1,500 amino acids, some proteins were modeled without signal peptides or C-terminal domains if these trimmings did not alter a predicted domain. The models were observed and superimposed in PyMol (Schrödinger 2010).

\section{Mimetic proteins detection in additional pathosystems.}

To test the power of detection of the pipeline to detect proteins with mimetic roles, we explored additional plant-pathogen pathosystems. In that sense, we decided to use two well-documented cases of molecular mimicry: RALF peptides of $F$. oxysporum and CLE from $H$. glycines. We reproduced the BLAST searches but changed the proteomes for the comparison (SBP and SBH).

In the case of RALF, we used the proteome of $F$. oxysporum f. sp. lycopersici (UP000009097) plus the sequence of RALF (A0A0J9VUZ1) that was not included in the proteomic file downloaded from UniProt database (downloaded 23 January 2021). The other proteomes used in this analysis are presented in the Supplementary Table S4.

To detect CLE proteins, we used 316 proteins of $H$. glycines available in UniProt, including HgCLE1 (Q9BN21) and HgCLE2 (Q86RQ1). As a host, we decide to use A. thaliana instead of soybean (the natural host of $H$. glycines) for the level of annotation in the proteome. In any case, $H$. glycines had the ability to infect $A$. thaliana in experimental conditions (Wang et al. 2010; Wang et al. 2005). The rest of the proteomes used in this analysis are presented in the Supplementary Table S5.

\section{Web server implementation.}

To allow the utilization of the pipeline described in this work, we developed a Web server called Molecular Mimicry Finder with the first steps of the methodology described above. The web server downloads the proteomes provided by the user, then makes the BLAST searches (SBP and SBH), and, finally, provides the subcellular localization for the pairs of candidates (imitator and imitated) with the higher bit score differences. The user must provide the UniProt proteome ID (e.g., UP000006548) for the host, the pathogen, and a group of species phylogenetically like the pathogen but with a different lifestyle (between 5 and 15 species is recommended). Additionally, the user can change the factor that multiplies the interquartile range for SBP (0 by default) and $\mathrm{SBH}$ (1.5 by default). This factor only affects the number of results shown in the final set of results with higher bit score differences. For example, if the user changes the factor for 1.5 , the algorithm will multiply 1.5 for the interquartile range of the differences of bit scores of all of the candidates obtained by BLAST. Then, only the candidates over 1.5 interquartile range (outliers) will be presented, and their subcellular localization shown. The presence of the subcellular localization information for the candidates depends on the level of annotation available in UniProt database. At the end of the process, the user will receive four files by email: the complete list of candidates detected by SBP (pathogen candidates) and SBH (host candidates) and the best results using the interquartile range criteria for SBP (pathogen_search_best.tsv) and SBH (host_search_best.tsv) with the subcellular localization annotation. 


\section{ACKNOWLEDGMENTS}

We thank K. Jaramillo, S. Solórzano, and K. Jimenes for their valuable assistance in the development of this work.

\section{AUTHOR-RECOMMENDED INTERNET RESOURCES}

Molecular Mimicry Finder:

https://bioquimio.udla.edu.ec/molecular-mimicry

EnsemblFungi Ustilago maydis database:

https://fungi.ensembl.org/Ustilago_maydis/Info/Index

Gene Expression Omnibus database: https://www.ncbi.nlm.nih.gov/geo

NCBI: https://www.ncbi.nlm.nih.gov/

UniProt: https://www.uniprot.org/

\section{LITERATURE CITED}

Alto, N. M., and Dixon, J. E. 2008. Analysis of Rho-GTPase mimicry by a family of bacterial type III effector proteins. Methods Enzymol. 439:131-143.

Alto, N. M., Shao, F., Lazar, C. S., Brost, R. L., Chua, G., Mattoo, S., McMahon, S. A., Ghosh, P., Hughes, T. R., Boone, C., and Dixon, J. E. 2006. Identification of a bacterial type III effector family with G protein mimicry functions. Cell 124:133-145.

Altschul, S. F., Madden, T. L., Schäffer, A. A., Zhang, J., Zhang, Z., Miller, W., and Lipman, D. J. 1997. Gapped BLAST and PSI-BLAST: A new generation of protein database search programs. Nucleic Acids Res. 25:3389-3402.

Anders, S., and Huber, W. 2010. Differential expression analysis for sequence count data. Genome Biol. 11:R106.

Ansari Qeshmi, S., Dabbagh, F., Borhani Haghighi, A., and Ghasemi, Y. 2016. Bioinformatics evaluation of the possibility of heat shock proteins as autoantigens in multiple sclerosis based on molecular mimicry hypothesis. J. Neuroimmunol. 295-296:100-121.

Armijos-Jaramillo, V. 2014. Trasferencia horizontal de genes y mimetismo molecular en hongos fitopatógenos del género Colletotrichum. Thesis, Universidad de Salamanca.

Bhardwaj, T., Haque, S., and Somvanshi, P. 2018. In silico identification of molecular mimics involved in the pathogenesis of Clostridium botulinum ATCC 3502 strain. Microb. Pathog. 121:238-244.

Blanco-Herrera, F., Moreno, A. A., Tapia, R., Reyes, F., Araya, M., D'Alessio, C., Parodi, A., and Orellana, A. 2015. The UDP-glucose: Glycoprotein glucosyltransferase (UGGT), a key enzyme in ER quality control, plays a significant role in plant growth as well as biotic and abiotic stress in Arabidopsis thaliana. BMC Plant Biol. 15:127.

Brefort, T., Doehlemann, G., Mendoza-Mendoza, A., Reissmann, S., Djamei, A., and Kahmann, R. 2009. Ustilago maydis as a pathogen. Annu. Rev. Phytopathol. 47:423-445.

Breitsprecher, D., and Goode, B. L. 2013. Formins at a glance. J. Cell Sci. 126:1-7.

Chan, S. L., Low, L. Y., Hsu, S., Li, S., Liu, T., Santelli, E., Le Negrate, G., Reed, J. C., Woods, V. L., Jr., and Pascual, J. 2009. Molecular mimicry in innate immunity: Crystal structure of a bacterial TIR domain. J. Biol. Chem. 284:21386-21392.

Chen, S., Lang, P., Chronis, D., Zhang, S., De Jong, W. S., Mitchum, M. G., and Wang, X. 2015. In planta processing and glycosylation of a nematode CLAVATA3/ENDOSPERM SURROUNDING REGIONlike effector and its interaction with a host CLAVATA2-like receptor to promote parasitism. Plant Physiol. 167:262-272.

Christensen, S. A., and Kolomiets, M. V. 2011. The lipid language of plant-fungal interactions. Fungal Genet. Biol. 48:4-14.

Clark, S. M., Running, M. P., and Meyerowitz, E. M. 1995. CLAVATA3 is a specific regulator of shoot and floral meristem development affecting the same processes as CLAVATA1. Development 121:2057-2067.

Damian, R. T. 1964. Molecular mimicry: Antigen sharing by parasite and host and its consequences. Am. Nat. 98:129-149.

Djamei, A., and Kahmann, R. 2012. Ustilago maydis: Dissecting the molecular interface between pathogen and plant. PLoS Pathog. 8:e1002955.

Doxey, A. C., and McConkey, B. J. 2013. Prediction of molecular mimicry candidates in human pathogenic bacteria. Virulence 4:453-466.

Drayman, N., Glick, Y., Ben-nun-shaul, O., Zer, H., Zlotnick, A., Gerber, D., Schueler-Furman, O., and Oppenheim, A. 2013. Pathogens use structural mimicry of native host ligands as a mechanism for host receptor engagement. Cell Host Microbe 14:63-73.
Elde, N. C., and Malik, H. S. 2009. The evolutionary conundrum of pathogen mimicry. Nat. Rev. Microbiol. 7:787-797.

Galán, J. E., and Zhou, D. 2000. Striking a balance: Modulation of the actin cytoskeleton by Salmonella. Proc. Natl. Acad. Sci. U.S.A. 97:8754-8761.

Gangappa, S., van Dyk, L. F., Jewett, T. J., Speck, S. H., and Virgin, H. W., 4th. 2002. Identification of the in vivo role of a viral bcl-2. J. Exp. Med. 195:931-940.

Guindon, S., Dufayard, J.-F., Lefort, V., Anisimova, M., Hordijk, W., and Gascuel, O. 2010. New algorithms and methods to estimate maximum-likelihood phylogenies: Assessing the performance of PhyML 3.0. Syst. Biol. 59:307-321.

Guo, Y., Ni, J., Denver, R., Wang, X., and Clark, S. E. 2011. Mechanisms of molecular mimicry of plant CLE peptide ligands by the parasitic nematode Globodera rostochiensis. Plant Physiol. 157:476-484.

Hall, R. 1994. Molecular mimicry. Page 314 in: Advances in Parasitology. J. R. Baker, R., Muller, and D. Rollinson, eds. Academic Press, Cambridge, MA, U.S.A.

Hebert, F. O., Phelps, L., Samonte, I., Panchal, M., Grambauer, S. Barber, I., Kalbe, M., Landry, C. R., and Aubin-Horth, N. 2015. Identification of candidate mimicry proteins involved in parasite-driven phenotypic changes. Parasit. Vectors 8:225.

Heger, A., and Holm, L. 2000. Rapid automatic detection and alignment of repeats in protein sequences. Proteins 41:224-237.

Horton, P., Park, K.-J., Obayashi, T., Fujita, N., Harada, H., Adams-Collier, C. J., and Nakai, K. 2007. WoLF PSORT: Protein localization predictor Nucleic Acids Res. 35:W585-W587.

Hunter, S., Jones, P., Mitchell, A., Apweiler, R., Attwood, T. K., Bateman, A., Bernard, T., Binns, D., Bork, P., Burge, S., de Castro, E., Coggill, P., Corbett, M., Das, U., Daugherty, L., Duquenne, L., Finn, R. D. Fraser, M., Gough, J., Haft, D., Hulo, N., Kahn, D., Kelly, E., Letunic, I., Lonsdale, D., Lopez, R., Madera, M., Maslen, J., McAnulla, C., McDowall, J., McMenamin, C., Mi, H., Mutowo-Muellenet, P., Mulder, N., Natale, D., Orengo, C., Pesseat, S., Punta, M., Quinn, A. F. Rivoire, C., Sangrador-Vegas, A., Selengut, J. D., Sigrist, C. J. A., Scheremetjew, M., Tate, J., Thimmajanarthanan, M., Thomas, P. D. Wu, C. H., Yeats, C., and Yong, S.-Y. 2012. InterPro in 2011: New developments in the family and domain prediction database. Nucleic Acids Res. 40:D306-D312.

Kamoun, S. 2007. Groovy times: Filamentous pathogen effectors revealed. Curr. Opin. Plant Biol. 10:358-365.

Kato, T., Watanabe, N., Morishima, Y., Fujita, A., Ishizaki, T., and Narumiya, S. 2001. Localization of a mammalian homolog of diaphanous, mDia1, to the mitotic spindle in HeLa cells. J. Cell Sci. 114:775-784.

Katoh, K., and Standley, D. M. 2013. MAFFT multiple sequence alignment software version 7: Improvements in performance and usability. Mol. Biol. Evol. 30:772-780.

Kelley, L. A., Mezulis, S., Yates, C. M., Wass, M. N., and Sternberg, M. J. E. 2015. The Phyre2 web portal for protein modeling, prediction and analysis. Nat. Protoc. 10:845-858.

Klink, B. U., Barden, S., Heidler, T. V., Borchers, C., Ladwein, M., Stradal, T. E. B., Rottner, K., and Heinz, D. W. 2010. Structure of Shigella IpgB2 in complex with human RhoA: Implications for the mechanism of bacterial guanine nucleotide exchange factor mimicry. J. Biol. Chem. 285:17197-17208.

Koeck, M., Hardham, A. R., and Dodds, P. N. 2011. The role of effectors of biotrophic and hemibiotrophic fungi in infection. Cell. Microbiol. 13:1849-1857.

Krogh, A., Larsson, B., von Heijne, G., and Sonnhammer, E. L. 2001. Predicting transmembrane protein topology with a hidden Markov model: Application to complete genomes. J. Mol. Biol. 305:567-580.

Kubrycht, J., and Novotná, J. 2014. Sequence-based prediction of linear autoepitopes involved in pathogenesis of IPAH and the corresponding organism sources of molecular mimicry. Int. J. Bioinf. Res. Appl. 10:587-612.

Kühn, S., and Geyer, M. 2014. Formins as effector proteins of Rho GTPases. Small GTPases 5:e29513.

Kunze, M., and Berger, J. 2015. The similarity between N-terminal targeting signals for protein import into different organelles and its evolutionary relevance. Front. Physiol. 6:259.

Lanver, D., Müller, A. N., Happel, P., Schweizer, G., Haas, F. B., Franitza, M., Pellegrin, C., Reissmann, S., Altmüller, J., Rensing, S. A., and Kahmann, R. 2018. The biotrophic development of Ustilago maydis studied by RNA-seq analysis. Plant Cell 30:300-323.

Lanver, D., Tollot, M., Schweizer, G., Lo Presti, L., Reissmann, S., Ma, L.-S., Schuster, M., Tanaka, S., Liang, L., Ludwig, N., and Kahmann, R. 2017. Ustilago maydis effectors and their impact on virulence. Nat. Rev. Microbiol. 15:409-421. 
Leslie, N. R., and Downes, C. P. 2002. PTEN: The down side of PI 3-kinase signalling. Cell. Signal. 14:285-295.

Loshaj-Shala, A., Regazzoni, L., Daci, A., Orioli, M., Brezovska, K., Panovska, A. P., Beretta, G., and Suturkova, L. 2015. Guillain Barré syndrome (GBS): New insights in the molecular mimicry between C. jejuni and human peripheral nerve (HPN) proteins. J. Neuroimmunol. 289:168-176.

Ludin, P., Nilsson, D., and Mäser, P. 2011. Genome-wide identification of molecular mimicry candidates in parasites. PLoS One 6:e17546.

Luo, Q., Pagel, P., Vilne, B., and Frishman, D. 2011. DIMA 3.0: Domain Interaction Map. Nucleic Acids Res. 39:D724-D729.

Masachis, S., Segorbe, D., Turrà, D., Leon-Ruiz, M., Fürst, U., El Ghalid, M., Leonard, G., López-Berges, M. S., Richards, T. A., Felix, G., and Di Pietro, A. 2016. A fungal pathogen secretes plant alkalinizing peptides to increase infection. Nat. Microbiol. 1:16043.

McArt, S. H., Miles, T. D., Rodriguez-Saona, C., Schilder, A., Adler, L. S., and Grieshop, M. J. 2016. Floral scent mimicry and vectorpathogen associations in a pseudoflower-inducing plant pathogen system. PLoS One 11:e0165761.

Mitchum, M. G., Wang, X., Wang, J., and Davis, E. L. 2012. Role of nematode peptides and other small molecules in plant parasitism. Annu. Rev. Phytopathol. 50:175-195.

Múnera, M., Farak, J., Pérez, M., Rojas, J., Villero, J., Sánchez, A., Sánchez, J., and Emiliani, Y. 2020. Prediction of molecular mimicry between antigens from Leishmania sp. and human: Implications for autoimmune response in systemic lupus erythematosus. Microb. Pathog. 148:104444.

Ngugi, H. K., and Scherm, H. 2006. Mimicry in plant-parasitic fungi. FEMS Microbiol. Lett. 257:171-176.

Olsen, A. N., and Skriver, K. 2003. Ligand mimicry? Plant-parasitic nematode polypeptide with similarity to CLAVATA3. Trends Plant Sci. 8:55-57.

Petersen, T. N., Brunak, S., von Heijne, G., and Nielsen, H. 2011. SignalP 4.0: Discriminating signal peptides from transmembrane regions. Nat. Methods 8:785-786.

Petre, B., Lorrain, C., Saunders, D. G. O., Win, J., Sklenar, J., Duplessis, S., and Kamoun, S. 2016. Rust fungal effectors mimic host transit peptides to translocate into chloroplasts. Cell. Microbiol. 18:453-465.

Popoff, M. R. 2014. Bacterial factors exploit eukaryotic Rho GTPase signaling cascades to promote invasion and proliferation within their host. Small GTPases 5:e28209.

Portnyagina, O., Zelepuga, E., Khomenko, V., Solov'eva, E., Solov'eva, T., and Novikova, O. 2018. In silico and in vitro analysis of cross-reactivity between Yersinia pseudotuberculosis OmpF porin and thyroidstimulating hormone receptor. Int. J. Biol. Macromol. 107:2484-2491.

Rafiqi, M., Jelonek, L., Akum, N. F., Zhang, F., and Kogel, K.-H. 2013. Effector candidates in the secretome of Piriformospora indica, a ubiquitous plant-associated fungus. Front. Plant Sci. 4:228.
Rawlings, N. D., Barrett, A. J., and Finn, R. 2016. Twenty years of the MEROPS database of proteolytic enzymes, their substrates and inhibitors. Nucleic Acids Res. 44:D343-D350.

R Development Core Team. 2011. R: A Language and Environment for Statistical Computing. R Foundation for Statistical Computing, Vienna, Austria.

Schrödinger LLC. 2010. The PyMOL Molecular Graphics System, Version 1.3r1. Schrödinger LLC, New York, NY, U.S.A.

Shahrizaila, N., and Yuki, N. 2011. Guillain-barré syndrome animal model: The first proof of molecular mimicry in human autoimmune disorder. J. Biomed. Biotechnol. 2011:829129.

Shankar, A., Agrawal, N., Sharma, M., Pandey, A., and Pandey, G. K. 2015. Role of protein tyrosine phosphatases in plants. Curr. Genomics 16:224-236.

Song, J.-H., Wei, W., Lv, B., Lin, Y., Yin, W.-X., Peng, Y.-L., Schnabel, G., Huang, J.-B., Jiang, D.-H., and Luo, C.-X. 2016. Rice false smut fungus hijacks the rice nutrients supply by blocking and mimicking the fertilization of rice ovary. Environ. Microbiol. 18:38403849.

Stebbins, C. E., and Galán, J. E. 2001. Structural mimicry in bacterial virulence. Nature 412:701-705.

Takeya, R., and Sumimoto, H. 2003. Fhos, a mammalian formin, directly binds to $\mathrm{F}$-actin via a region $\mathrm{N}$-terminal to the $\mathrm{FH} 1$ domain and forms a homotypic complex via the $\mathrm{FH} 2$ domain to promote actin fiber formation. J. Cell Sci. 116:4567-4575.

Tholl, S., Moreau, F., Hoffmann, C., Arumugam, K., Dieterle, M., Moes, D., Neumann, K., Steinmetz, A., and Thomas, C. 2011. Arabidopsis actin-depolymerizing factors (ADFs) 1 and 9 display antagonist activities. FEBS Lett. 585:1821-1827.

Urban, M., Cuzick, A., Rutherford, K., Irvine, A., Pedro, H., Pant, R. Sadanadan, V., Khamari, L., Billal, S., Mohanty, S., and Hammond-Kosack, K. E. 2017. PHI-base: A new interface and further additions for the multispecies pathogen-host interactions database. Nucleic Acids Res. 45:D604D610.

Wang, J., Lee, C., Replogle, A., Joshi, S., Korkin, D., Hussey, R., Baum, T. J., Davis, E. L., Wang, X., and Mitchum, M. G. 2010. Dual roles for the variable domain in protein trafficking and host-specific recognition of Heterodera glycines CLE effector proteins. New Phytol. 187:1003-1017.

Wang, J., Replogle, A., Hussey, R., Baum, T., Wang, X., Davis, E. L., and Mitchum, M. G. 2011. Identification of potential host plant mimics of CLAVATA3/ESR (CLE)-like peptides from the plant-parasitic nematode Heterodera schachtii. Mol. Plant Pathol. 12:177-186.

Wang, X., Mitchum, M. G., Gao, B., Li, C., Diab, H., Baum, T. J., Hussey, R. S., and Davis, E. L. 2005. A parasitism gene from a plantparasitic nematode with function similar to CLAVATA3/ESR (CLE) of Arabidopsis thaliana. Mol. Plant Pathol. 6:187-191. 